\title{
Formation of a Plasma Conductor by Induction
}

\author{
Ryan van Herel*, Rowan Sinton, Wade Enright, Pat Bodger \\ Department of Electrical and Electronic Engineering \\ University of Canterbury \\ Christchurch, New Zealand \\ Email: *ryan.vanherel@pg.canterbury.ac.nz
}

\begin{abstract}
Plasma has been formed by exploding a wire in an inductively coupled circuit. Capacitors are discharged through air-cored mutually coupled coils. The exploding wire section of the electrically isolated coil is $0.2 \mathrm{~mm}$ diameter copper wire. The energy source is a $21.4 \mu F$ capacitor bank, charged to $21.5 \mathrm{kV}$, producing $0.5 \mathrm{~m}$ of plasma discharge in a single turn. Waveforms of voltage and current for the energy source are presented, as well as waveforms of the exploding wire voltage. There are some discussions on the electrical characteristics and the copper residues left behind from the wire explosion.
\end{abstract}

\section{INTRODUCTION}

Exploding wires (EW) are presently being researched at the University of Canterbury. In one mode of experimentation, a high voltage pulse is applied directly to the wire from a high-power electrical source, usually a capacitor bank. This has been the principal method of instigating wire explosion in various studies to date [1] [2] [3]. All such investigations describe direct conduction of high voltage impulse to the exploding wire. Another experimental mode of EW is under investigation, namely that of producing exploding wire plasmas using electromagnetic induction. To date, no such attempt has been made to create exploding wire plasmas by induction by other researchers, and as a result, there is no literature in this area. The ultimate intention of the University of Canterbury experiments is to produce a ring of plasma which is electrically isolated and only magnetically coupled to the capacitor-side circuit. The exploding wire plasma expands as it ionises. Once a full turn of plasma has formed, the expansion of the plasma conductor's diameter will cause it to short out at its start and end points, resulting in the plasma ring desired.

The wire may undergo two types of explosion in experiments described herein. One type is where the wire forms a copper vapour and some limited ionisation may occur within this cloud of particles. The other type is where the wire is vaporised and there is sufficient uniform ionisation to form a plasma discharge channel. In the latter case, the wire is said to have produced a restrike. The various combinations of plasma and copper gas produced in the wire explosion can condense or settle onto a robust paper or plastic surface, producing particular patterns of residue.

For a device transferring energy via magnetic induction, there are many arrangements of conductor that can be conceived of, for instance: pancake coils, helical coils, disk coils or layer windings. Many winding set configurations

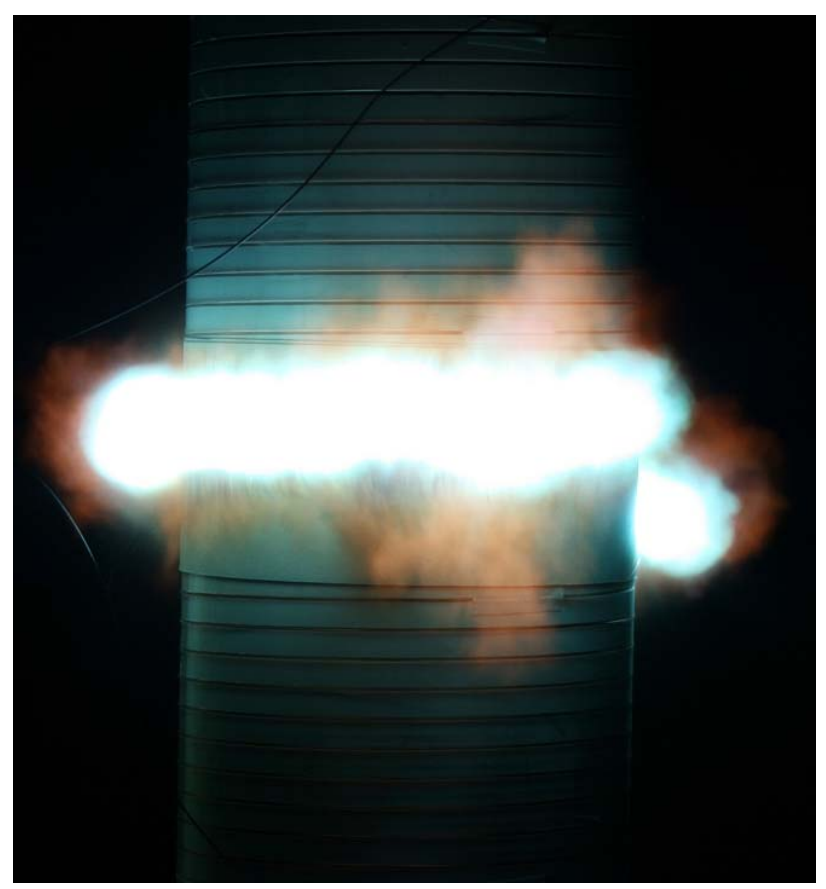

Fig. 1. A complete 360 degree turn of plasma produced by induction.

were experimented with, all producing some partial plasma formation or EW effect. This paper describes an experiment of high voltage capacitor discharge into mutually coupled coils, which facilitated the explosion of half a metre of solid copper wire into a plasma turn, shown in fig. 1 . The apparatus is described in detail. Waveforms are presented, illustrating certain electrical characteristics of the inductively produced wire explosion.

\section{EQUIPMENT}

The circuit used in this set of experiments is depicted in fig. 2. It consists of a $21.4 \mu \mathrm{F}$, high voltage capacitor bank, $C$, (comprised of 20 oil-insulated capacitors) connected via a three-electrode triggered spark-gap (TSG) to air-cored mutually coupled coils, $L_{1}$ and $L_{2}$. The mutually coupled coils have two sets of concentric windings arranged in a helical configuration, shown in fig. 3 ; the inside winding is $L_{1}$ and is connected to the capacitor bank, and the outside winding consists of the two receiver coils $L_{2}$ and the exploding wire $(E W)$. The $L_{1}$ winding diameter is $140 \mathrm{~mm}$, and is wound from 20 turns of $2 \mathrm{~mm}$ diameter, enamelled copper wire. The 


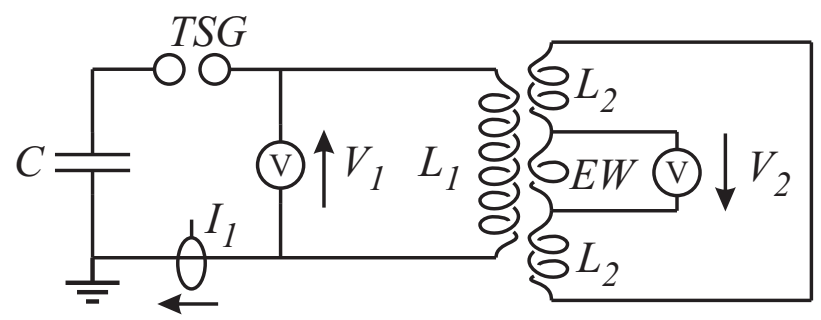

Fig. 2. Circuit used for creating EW by induction.

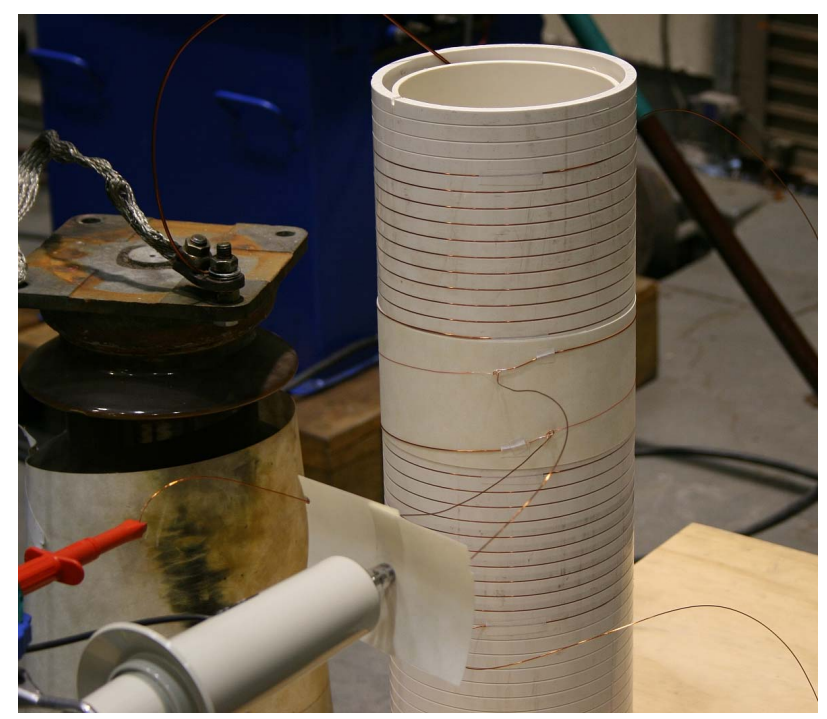

Fig. 3. Mutually coupled coils set-up prior to experimentation, in the University of Canterbury high voltage laboratory. The outside winding consisting of the two receiver coils, and the exploding wire can be seen. The inside winding is obscured from view. The connection from the EW to the Tektronix high voltage probe can be seen in the foreground

outside winding is $160 \mathrm{~mm}$ in diameter and is composed of two $0.63 \mathrm{~mm}$ diameter copper wire receiver coils of 10 turns each; with the exploding wire being 1 turn of $0.2 \mathrm{~mm}$ of polyamideenamelled copper wire connected between said receiver coils. The ends of the receiver coils are bared and twisted together so that the outside winding is in short circuit. All winding sets are wound in the same sense, onto polyvinylchloride (PVC) formers with grooves in them fabricated to a predetermined winding pitch. $L_{1}$ winding is of $18 \mathrm{~mm}$ pitch, while the receiver coils are wound with $11 \mathrm{~mm}$ pitch. The EW winding pitch is $56 \mathrm{~mm}$; this is enough clearance so that the terminals do not short out before the EW forms a restrike. The receiver coils are required in the outside winding to increase the flux linkages to this electrically isolated circuit. This increases the voltage applied to the EW.

The $V_{1}$ voltage was measured using a Ferranti $600 \mathrm{kV}$ capacitive divider and the $I_{1}$ current was measured with a Pearson $50 \mathrm{kA}$ ferrite-cored high frequency current transformer. The EW voltage, $V_{2}$ was measured using a Tektronix $40 \mathrm{kV}$ d.c. high voltage probe. Two separate battery-inverter sets were used as the isolated power supplies for the recording oscilloscopes. The first oscilloscope measured the voltage $V_{1}$ and current $I_{1}$, and the second oscilloscope measured the voltage $V_{2}$ across the EW.

\section{RESULTS}

\section{A. Electrical Characteristics}

Waveforms were obtained for an EW-by-induction experiment which produced a complete plasma turn, where the capacitor bank was charged to a voltage of $21.5 \mathrm{kV}$ d.c. The capacitor-side voltage, $V_{1}$, current, $I_{1}$, and also the voltage across the EW, $V_{2}$, were all recorded and are displayed in fig. 4 and fig. 5. Neglecting the $L \cdot d i / d t$ voltage component in the wire, the EW can be considered as a varying resistance, so by Ohm's law, it is possible to deduce the state of the wire at different parts of the waveform by looking carefully at the EW voltage. In voltage $V_{2}$ of fig. 5 , initially there is a large voltage spike which can be taken as the wire fragmentation stage. Following this is a period of time from $25 \mu s$ to $75 \mu s$ where the EW can be said to be an open circuit, so the open circuit voltage $V_{2}$ approximately follows the voltage $-V_{1}$. Following this, and once a restrike takes place, the continuous ionisation path has formed; the voltage collapses into a shallow distorted sinusoid pattern. The EW can be said to be in rudimentary short circuit - for there is still a load with some finite resistance due to the plasma. The exploding wire voltage spike appears to have a similar shape to those of short length EW experiments conducted with high circuit inductance [4].

Sinton describes a feature of EW restrike where plasma beads form along the axis of the wire and link together to form a plasma channel [5]. For the EW by induction, Sinton's plasma beads were not observed during experiments. The Authors believe that the EW plasma in the induction experiments are from a different restrike mechanism to that identified by Sinton. It is also worth noting that the applied induction voltages are oscillatory whereas the Sinton voltages are direct application of d.c. over similar time frames. A non-restriking inductive EW is shown in fig. 6. The orangecoloured incandescent copper vapour can be seen; this resembles Sinton's excessive average electric field outcome of wire explosion in [5].

The receiver coils may experience some mechanical distortion. These forces acting on these coils appear to be consistent with forces of the type experienced by conventional power transformers; namely a bursting force on the outer winding as well as an axial force that makes it spring toward its magnetic centre. On occasions where there is no formation of a continuous EW plasma discharge, i.e. non-restrike, the receiver coils will remain in place indicating that these coils experienced no conduction of large currents. On the other hand if a continuous EW plasma discharge forms, the receiver coils will simultaneously burst away from the outside former and collapse along the axis toward the device axial centre. This outcome is because the receiver coils have conducted the large EW discharge current and as such, it is taken as further evidence of EW plasma formation. 


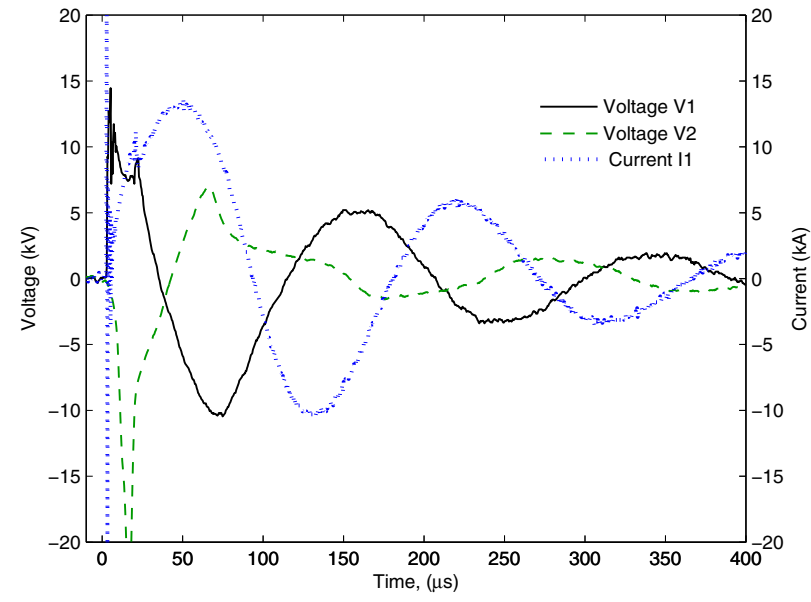

Fig. 4. $\quad V_{1}$ voltage, $I_{1}$ current and $V_{2}$ voltage for the EW-by-induction experiment producing restrike.

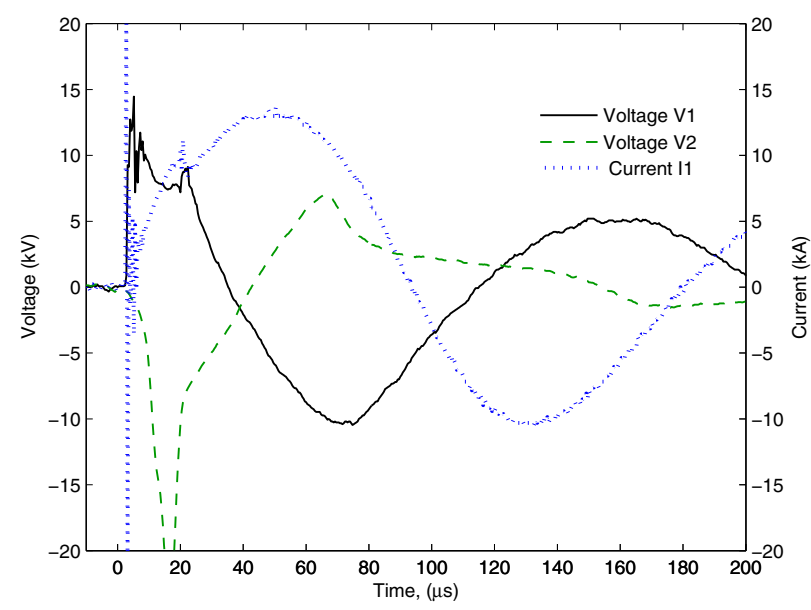

Fig. 5. First $200 \mu \mathrm{s}$ of $V_{1}$ voltage, $I_{1}$ current and $V_{2}$ voltage for the EWby-induction experiment producing restrike.

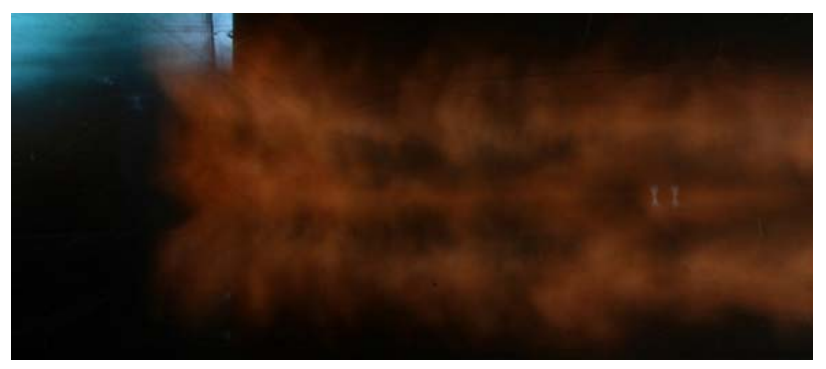

Fig. 6. Photograph of experiment producing non-restrike EW effect. Three EW turns around the PVC former.

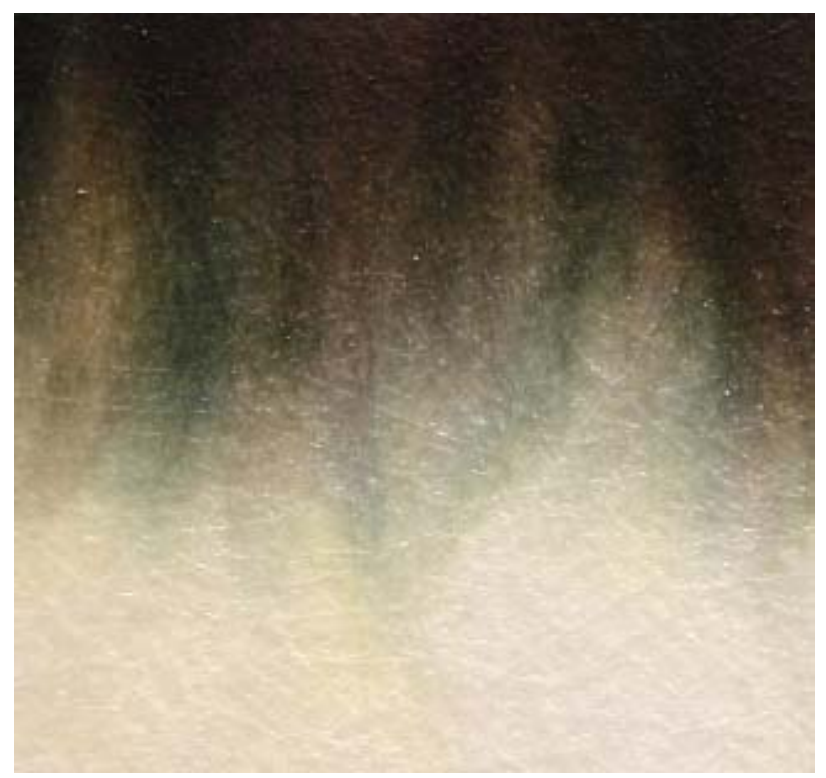

Fig. 7. Photograph of copper oxide residues left behind by wire explosion.

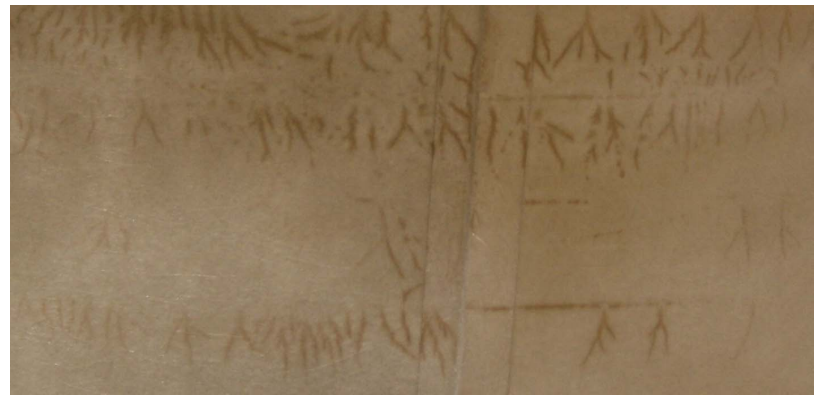

Fig. 8. Copper dust-trees on Nomex paper left behind after an EW-byinduction experiment.

\section{B. Residues}

The residues left behind by the EW are conductive and if they are allowed to condense on the PVC former's surface, will compromise the integrity of subsequent experiments by facilitating turn-to-turn flashovers on the receiver coils. Nomex insulating paper was used for keeping the copper EW residue and contaminants off the PVC former of the outside winding. The Nomex itself was also good for recording the type of EW explosion that had occurred. When an EW plasma turn has formed, it typically deposits a $60-70 \mathrm{~mm}$ wide band of explosion residue, depicted in fig. 7. The residue contains copper(I) and copper(II) oxides, but it is principally composed of black copper(II) oxide [6]. Where the EW plasma does not form, there will still be a band of residue containing predominantly copper(II) oxide, but this band is generally no wider than $50 \mathrm{~mm}$, and there are discontinuous residue patterns along the band. This evidence indicates that a continuous EW plasma has not formed.

Also observed were electrical dust-trees between the turns of the receiver coil, an example of which is shown in fig. 8. These dust-trees were formed in the presence of the copper 
vapour, some time during the EW event. It was possible to rub the dust trees off the surface of the paper or PVC after the experiment.

\section{CONCLUSION}

A full 360 degree $0.5 \mathrm{~m}$ long plasma turn has been created in the explosion of copper wire by electromagnetic induction. Waveforms and photography suggest this is a different mechanism of wire explosion to that of conventional capacitive discharges into straight wires. Coil condition and the quantity of copper oxide residues left behind from the EW can help inform experimenters about the magnitude of current and the outcome of the EW, i.e. whether it produced restrike.

\section{ACKNOWLEDGMENT}

The authors would like to acknowledge the support from J. Lawrence (Electric Power Engineering Centre) and technical support from D. Healy, K. Smart and J. Woudberg.

\section{REFERENCES}

[1] A. E. Vlastos, "Current pause in exploding-wire discharges," Journal of Applied Physics, vol. 38, no. 13, pp. 4993 -4998, dec 1967.

[2] F. D. Bennett, H. S. Burden, and D. D. Shear, "Correlated electrical and optical measurements of exploding wires," Physics of Fluids, vol. 5, no. 1, pp. 102-113, 1962.

[3] M. J. Taylor, "Interruption of the explosion of plasma initiator wires," in 12th Symposium on Electromagnetic Launch Technology, 2004.

[4] M. J. Taylor, "Formation of plasma around wire fragments created by electrically exploded copper wire," Journal of Physics D-Applied Physics, vol. 35, no. 7, pp. 700-709, 2002.

[5] R. Sinton, R. van Herel, W. Enright, and P. Bodger, "Observations of the long distance exploding wire restrike mechanism," Journal of Applied Physics, vol. 108, no. 5, p. 053304, 2010. [Online]. Available: http://link.aip.org/link/?JAP/108/053304/1

[6] M. J. Taylor, "Plasma propellant interactions in an electrothermalchemical gun," Ph.D. dissertation, Royal Military College of Science, Cranfield University, 2002 - 2003. 